\title{
Test the time-reversal modified universality of the Sivers function
}

\author{
Zhong-Bo Kang1, 团 and Jian-Wei Qiu, \\ ${ }^{1}$ Department of Physics and Astronomy, Iowa State University, Ames, IA 50011, USA
}

(Dated: November 1, 2018)

\begin{abstract}
We derive the time-reversal modified universality for both quark and gluon Sivers function from the parity and time-reversal invariance of QCD. We calculate the single transverse-spin asymmetry of inclusive lepton from the decay of $W$ bosons in polarized proton-proton collision at RHIC, in terms of the Sivers function. We find that although the asymmetry is diluted from the $W$ decay, the lepton asymmetry is at the level of several percent and is measurable for a good range of lepton rapidity at RHIC. We argue that this measurable lepton asymmetry at RHIC is an excellent observable for testing the time-reversal modified universality of the Sivers function.

PACS numbers: 12.38.Bx, 12.39.St, 13.85.Qk, 13.88+e
\end{abstract}

I. Introduction. Much of the predictive power of perturbative Quantum Chromodynamics (QCD) is contained in factorization theorems [1]. They normally include two assertions. One is that a physically measured quantity can be factorized into some perturbatively calculable short-distance hard parts convoluted with nonperturbative long-distance distribution functions (or matrix elements). The other is the universality of the nonperturbative distribution functions. Predictions follow when processes with different hard scatterings but the same distribution functions are compared. With one set of universal parton distribution functions (PDFs) the leading power collinear QCD factorization formalisms have been very successful in interpreting and predicting almost all existing data from high energy collisions with momentum transfers larger than a few $\mathrm{GeV}$ [2].

The phenomenon of single transverse-spin asymmetry $(\mathrm{SSA}), A_{N} \equiv\left(\sigma\left(\vec{S}_{\perp}\right)-\sigma\left(-\vec{S}_{\perp}\right)\right) /\left(\sigma\left(\vec{S}_{\perp}\right)+\sigma\left(-\vec{S}_{\perp}\right)\right)$, defined as the ratio of the difference and the sum of the cross sections when the single transverse spin vector $\vec{S}_{\perp}$ is flipped, was first observed in the hadronic $\Lambda^{0}$ production at Fermilab in 1976 as a surprise [3]. Large SSAs, as large as 30 percent, have been consistently observed in various experiments involving one polarized hadron at different collision energies [4]. The size of the SSAs presented a challenge to the leading power collinear QCD factorization formalism [5], and provided an excellent opportunity to probe a new domain of QCD dynamics.

Two widely discussed theoretical approaches have been proposed to evaluate the observed SSAs in QCD. One generalizes the QCD collinear factorization approach to the next-to-leading power in the momentum transfer [ 6 ], and attributes the SSA to the quantum interference of scattering amplitudes with different numbers of active partons [7, 8]. The size of the asymmetry is determined by new three-parton correlation functions 9 ]. This generalized collinear factorization approach is more relevant for the SSAs of cross sections whose momentum

\footnotetext{
*Electronic address: kangzb@iastate.edu
}

${ }^{\dagger}$ Electronic address: jwq@iastate.edu transfers $Q \gg \Lambda_{\mathrm{QCD}}$. The other approach factorizes the cross sections so as the SSAs in terms of the transverse momentum dependent (TMD) parton distributions 10, 11, 12, 13, 14], and attributes the SSAs to the Sivers function [15] (or the Collins function if a finalstate hadron was observed [16]). The TMD factorization approach is more suitable for the SSAs of cross sections with two very different momentum transfer scales, $Q_{1} \gg Q_{2} \gtrsim \Lambda_{\mathrm{QCD}}$. These two approaches each have their kinematic domain of validity, they were shown to be consistent with each other in the kinematic regime where they both apply [17].

However, there is one crucial difference between these two factorization approaches besides the difference in kinematic regimes where they apply. The Sivers function in the TMD factorization approach could be process dependent, while all distribution functions in the collinear factorization approach are universal. It was predicted by Collins [10] on the basis of time-reversal arguments that the quark Sivers function in semi-inclusive deep inelastic scattering (SIDIS) and in Drell-Yan process (DY) have the same functional form but an opposite sign, a time-reversal modified universality. In this Letter, we derive the same time-reversal modified universality for both quark and gluon Sivers function from the parity and time-reversal invariance of QCD.

The experimental check of this time-reversal modified universality of the Sivers function would provide a critical test for the TMD factorization approach [10, 11, 12, 13, 14]. Recently, the quark Sivers function has been extracted from data of SIDIS experiments [18]. Future measurements of the SSAs in DY production have been planned [19]. In this Letter, we present our calculation of the SSAs of inclusive single lepton production from the decay of $W$ bosons. The $W$ production and DY share the same Sivers function. We find that although the asymmetry is diluted from the decay of $W$ bosons, the lepton asymmetry is significant and measurable for a good range of lepton rapidity at RHIC. We show that the lepton SSAs provide the better flavor separation of the quark Sivers function than what the standard DY can do. We also show that the lepton SSAs are sharply peaked at transverse momentum $p_{T} \sim M_{W} / 2$ with $W$ mass $M_{W}$. 
Since leptons from heavy quarkonium decay and other potential backgrounds are unlikely to be peaked at the $p_{T} \sim M_{W} / 2$, we argue that the SSA of inclusive high $p_{T}$ leptons at RHIC is an excellent observable for testing the time-reversal modified universality of the Sivers function.

II. The QCD prediction. The predictive power of the TMD factorization approach to the SSAs relies on the universality of the TMD parton distributions. For the lepton-hadron SIDIS, $\ell(l)+h(p, \vec{S}) \rightarrow \ell^{\prime}\left(l^{\prime}\right)+h^{\prime}\left(p^{\prime}\right)+X$, the factorized TMD quark distribution has the following gauge invariant operator definition [20],

$$
\begin{aligned}
f_{q / h^{\uparrow}}^{\operatorname{SIDIS}}\left(x, \mathbf{k}_{\perp}, \vec{S}\right) & =\int \frac{d y^{-} d^{2} \mathbf{y}_{\perp}}{(2 \pi)^{3}} e^{i x p^{+} y^{-}-i \mathbf{k}_{\perp} \cdot \mathbf{y}_{\perp}} \\
& \times\langle p, \vec{S}| \bar{\psi}\left(0^{-}, \mathbf{0}_{\perp}\right) \Phi_{n}^{\dagger}\left(\{\infty, 0\}, \mathbf{0}_{\perp}\right) \\
& \times \Phi_{\mathbf{n}_{\perp}}^{\dagger}\left(\infty,\left\{\mathbf{y}_{\perp}, \mathbf{0}_{\perp}\right\}\right) \frac{\gamma^{+}}{2} \\
& \times \Phi_{n}\left(\left\{\infty, y^{-}\right\}, \mathbf{y}_{\perp}\right) \psi\left(y^{-}, \mathbf{y}_{\perp}\right)|p, \vec{S}\rangle
\end{aligned}
$$

where $y^{+}=0^{+}$dependence is suppressed and the gauge links from the final-state interaction of SIDIS are

$$
\begin{aligned}
\Phi_{n}\left(\left\{\infty, y^{-}\right\}, \mathbf{y}_{\perp}\right) & \equiv \mathcal{P} e^{-i g \int_{y^{-}}^{\infty} d y_{1}^{-} n^{\mu} A_{\mu}\left(y_{1}^{-}, \mathbf{y}_{\perp}\right)}, \\
\Phi_{\mathbf{n}_{\perp}}\left(\infty,\left\{\mathbf{y}_{\perp}, \mathbf{0}_{\perp}\right\}\right) & \equiv \mathcal{P} e^{-i g \int_{\mathbf{0}_{\perp}}^{\mathbf{y}_{\perp}} d \mathbf{y}_{\perp}^{\prime} \mathbf{n}_{\perp}^{\mu} A_{\mu}\left(\infty, \mathbf{y}_{\perp}^{\prime}\right)},
\end{aligned}
$$

where $\mathcal{P}$ indicates the path ordering and the direction $\mathbf{n}_{\perp}$ is pointed from $\mathbf{0}_{\perp}$ to $\mathbf{y}_{\perp}$. Here we define the lightcone vectors, $n^{\mu}=\left(n^{+}, n^{-}, \mathbf{n}_{\perp}\right)=\left(0,1, \mathbf{0}_{\perp}\right)$ and $\bar{n}^{\mu}=$ $\left(1,0, \mathbf{0}_{\perp}\right)$, which project out the light-cone components of any four-vector $V^{\mu}$ as $V \cdot n=V^{+}$and $V \cdot \bar{n}=V^{-}$.

For the DY, $h(p, \vec{S})+h^{\prime}\left(p^{\prime}\right) \rightarrow \gamma^{*}(Q)\left[\rightarrow \ell^{+} \ell^{-}\right]+X$, the factorized TMD quark distribution is given by

$$
\begin{aligned}
f_{q / h^{\uparrow}}^{\mathrm{DY}}\left(x, \mathbf{k}_{\perp}, \vec{S}\right) & =\int \frac{d y^{-} d^{2} \mathbf{y}_{\perp}}{(2 \pi)^{3}} e^{i x p^{+} y^{-}-i \mathbf{k}_{\perp} \cdot \mathbf{y}_{\perp}} \\
& \times\langle p, \vec{S}| \bar{\psi}\left(0^{-}, \mathbf{0}_{\perp}\right) \Phi_{n}^{\dagger}\left(\{-\infty, 0\}, \mathbf{0}_{\perp}\right) \\
& \times \Phi_{\mathbf{n}_{\perp}}^{\dagger}\left(-\infty,\left\{\mathbf{y}_{\perp}, \mathbf{0}_{\perp}\right\}\right) \frac{\gamma^{+}}{2} \\
& \times \Phi_{n}\left(\left\{-\infty, y^{-}\right\}, \mathbf{y}_{\perp}\right) \psi\left(y^{-}, \mathbf{y}_{\perp}\right)|p, \vec{S}\rangle
\end{aligned}
$$

where the past pointing gauge links were caused by the initial-state interactions of DY production [10]. From Eqs. (1) and (3), it is easy to show that the collinear quark distributions are process independent,

$$
\int d^{2} \mathbf{k}_{\perp} f_{q / h^{\uparrow}}^{\mathrm{SIDIS}}\left(x, \mathbf{k}_{\perp}, \vec{S}\right)=\int d^{2} \mathbf{k}_{\perp} f_{q / h^{\uparrow}}^{\mathrm{DY}}\left(x, \mathbf{k}_{\perp}, \vec{S}\right),
$$

if the same renormalization scheme was used for the ultraviolet divergence of the $\mathbf{k}_{\perp}$ integration.

Let $|\alpha\rangle=|p, \vec{S}\rangle$ and $\langle\beta|$ be equal to the rest of the matrix element in Eq. (1) [9]. From the parity and time-reversal invariance of QCD, $\left\langle\alpha_{P} \mid \beta_{P}\right\rangle=\langle\alpha \mid \beta\rangle$ and $\left\langle\beta_{T} \mid \alpha_{T}\right\rangle=\langle\alpha \mid \beta\rangle$, where $\left|\alpha_{P}\right\rangle$ and $\left|\beta_{P}\right\rangle$, and $\left|\alpha_{T}\right\rangle$ and $\left|\beta_{T}\right\rangle$ are the parity and time-reversal transformed states from the states $|\alpha\rangle$ and $|\beta\rangle$, respectively, we derive

$$
f_{q / h^{\uparrow}}^{\mathrm{SIDIS}}\left(x, \mathbf{k}_{\perp}, \vec{S}\right)=f_{q / h^{\uparrow}}^{\mathrm{DY}}\left(x, \mathbf{k}_{\perp},-\vec{S}\right) .
$$

and conclude that the spin-averaged TMD quark distributions are process independent. Following the notation of Ref. [18], we expand the TMD quark distribution as

$$
\begin{aligned}
f_{q / h^{\uparrow}}\left(x, \mathbf{k}_{\perp}, \vec{S}\right) & \equiv f_{q / h}\left(x, k_{\perp}\right) \\
& +\frac{1}{2} \Delta^{N} f_{q / h^{\uparrow}}\left(x, k_{\perp}\right) \vec{S} \cdot\left(\hat{p} \times \hat{\mathbf{k}}_{\perp}\right)
\end{aligned}
$$

where $k_{\perp}=\left|\mathbf{k}_{\perp}\right|, \hat{p}$ and $\hat{\mathbf{k}}_{\perp}$ are the unit vectors of $\vec{p}$ and $\mathbf{k}_{\perp}$, respectively, $f_{q / h}\left(x, k_{\perp}\right)$ is the spin-averaged TMD distribution, and $\Delta^{N} f_{q / h \uparrow}\left(x, k_{\perp}\right)$ is the Sivers function [15]. Substituting Eq. (6) into Eq. (5), we obtain,

$$
\Delta^{N} f_{q / h^{\uparrow}}^{\mathrm{SIDIS}}\left(x, k_{\perp}\right)=-\Delta^{N} f_{q / h^{\uparrow}}^{\mathrm{DY}}\left(x, k_{\perp}\right),
$$

which confirms the Collins' prediction [10] that the Sivers function in SIDIS and in DY differ by a sign.

We define the gauge invariant TMD gluon distribution in SIDIS and in DY by replacing the quark operator $\bar{\psi}\left(\gamma^{+} / 2\right) \psi$ in Eqs. (11) and (3) by the gluon operator $F^{+\mu} F^{+\nu}\left(-g_{\mu \nu}\right)$, and the gauge links by those in the adjoint representation of $\mathrm{SU}(3)$ color. From the parity and time-reversal invariance of the matrix elements of the TMD gluon distribution, we find, like Eq. (5),

$$
f_{g / h^{\uparrow}}^{\mathrm{SIDIS}}\left(x, \mathbf{k}_{\perp}, \vec{S}\right)=f_{g / h^{\uparrow}}^{\mathrm{DY}}\left(x, \mathbf{k}_{\perp},-\vec{S}\right) .
$$

Applying Eq. (6) to the gluon TMD distribution, we derive the same time-reversal modified universality for the gluon Sivers function,

$$
\Delta^{N} f_{g / h \uparrow}^{\operatorname{SIDIS}}\left(x, k_{\perp}\right)=-\Delta^{N} f_{g / h \uparrow}^{\mathrm{DY}}\left(x, k_{\perp}\right) .
$$

The sign change of the Sivers function is a property of the gauge invariant TMD parton distributions.

III. Lepton SSAs from $W$ production. The SSAs of $W$ production at RHIC, $A\left(p_{A}, \vec{S}_{\perp}\right)+B\left(p_{B}\right) \rightarrow W^{ \pm}(q) \rightarrow$ $\ell^{ \pm}(p)+X$, were proposed in Refs. 21] to measure the Sivers function. However, it is difficult to reconstruct $W$ bosons by the current detectors at RHIC. We present here our predictions for the SSAs of inclusive lepton production from the decay of $W$ bosons. We also present the SSAs of $W$ production for a comparison.

We use the TMD factorization formalism because $W$ bosons at RHIC are likely produced with transverse momentum $\left|\mathbf{q}_{\perp}\right| \ll M_{W}$. We work in a frame in which the polarized hadron $A$ moves in the $+z$-direction. We have the leading order spin-averaged $W$ cross section

$$
\begin{aligned}
\frac{d \sigma_{A B \rightarrow W}}{d y_{W} d^{2} \mathbf{q}_{\perp}}= & \sigma_{0} \sum_{a, b}\left|V_{a b}\right|^{2} \int d^{2} \mathbf{k}_{a \perp} d^{2} \mathbf{k}_{b \perp} f_{a / A}\left(x_{a}, k_{a \perp}\right) \\
& \times f_{b / B}\left(x_{b}, k_{b \perp}\right) \delta^{2}\left(\mathbf{q}_{\perp}-\mathbf{k}_{a \perp}-\mathbf{k}_{b \perp}\right),(10)
\end{aligned}
$$

where $y_{W}$ is the $W$ rapidity, $\sigma_{0}=(\pi / 3) \sqrt{2} \mathrm{G}_{\mathrm{F}} M_{W}^{2} / s$ is the lowest order partonic cross section with the Fermi weak coupling constant $\mathrm{G}_{F}$ and $s=\left(p_{A}+p_{B}\right)^{2}, \sum_{a b}$ runs over all light (anti)quark flavors, $V_{a b}$ are the CKM 
matrix elements for the weak interaction. The parton momentum fractions in Eq. (10) are given by

$$
x_{a}=\frac{M_{W}}{\sqrt{s}} e^{y_{W}}, \quad x_{b}=\frac{M_{W}}{\sqrt{s}} e^{-y_{W}}
$$

to the leading power in $q_{\perp}^{2} / M_{W}^{2}$. Similarly, we have the leading order factorized spin-dependent $W$ cross section $\Delta \sigma\left(\vec{S}_{\perp}\right)=\left[\sigma\left(\vec{S}_{\perp}\right)-\sigma\left(-\vec{S}_{\perp}\right)\right] / 2$ as

$$
\begin{aligned}
\frac{d \Delta \sigma_{A^{\uparrow} B \rightarrow W}\left(\vec{S}_{\perp}\right)}{d y_{W}} & d^{2} \mathbf{q}_{\perp} \\
& \times \vec{S}_{\perp} \cdot\left(\hat{p}_{A} \times \hat{\mathbf{k}}_{a \perp}\right) \Delta_{a, b}\left|V_{a b}\right|^{2} \int d_{a / A^{\uparrow}}^{\mathrm{DY}}\left(x_{a}, k_{a \perp}\right) \\
& \times f_{b / B}\left(x_{b}, k_{b \perp}\right) \delta^{2}\left(\mathbf{q}_{\perp \perp}-\mathbf{k}_{a \perp}-\mathbf{k}_{b \perp}\right) .
\end{aligned}
$$

The SSA of $W$ production is then defined as,

$$
A_{N}^{(W)} \equiv \frac{d \Delta \sigma\left(\vec{S}_{\perp}\right)_{A^{\uparrow} B \rightarrow W}}{d y_{W} d^{2} \mathbf{q}_{\perp}} / \frac{d \sigma_{A B \rightarrow W}}{d y_{W} d^{2} \mathbf{q}_{\perp}},
$$

whose sign depends on the sign of the Sivers function and the direction of the spin vector $\vec{S}_{\perp}$.

To evaluate the SSA in Eq. (13), we use the parameterization of TMD parton distributions in Ref. [18],

$$
\begin{aligned}
f_{q / h}\left(x, k_{\perp}\right) & =f_{q}(x) \frac{1}{\pi\left\langle k_{\perp}^{2}\right\rangle} e^{-k_{\perp}^{2} /\left\langle k_{\perp}^{2}\right\rangle}, \\
\Delta^{N} f_{q / h^{\uparrow}}^{\text {SIDIS }}\left(x, k_{\perp}\right) & =2 \mathcal{N}_{q}(x) h\left(k_{\perp}\right) f_{q / h}\left(x, k_{\perp}\right), \\
h\left(k_{\perp}\right) & =\sqrt{2 e} \frac{k_{\perp}}{M_{1}} e^{-k_{\perp}^{2} / M_{1}}
\end{aligned}
$$

where $f_{q}(x)$ is the standard unpolarized parton distribution of flavor $q,\left\langle k_{\perp}^{2}\right\rangle$ and $M_{1}$ are fitting parameters, and $\mathcal{N}_{q}(x)$ is a fitted distribution given in Ref. [18]. By carrying out the integration $d^{2} \mathbf{k}_{a \perp} d^{2} \mathbf{k}_{b \perp}$ in Eqs. (10) and (12) analytically, we obtain,

$$
\begin{aligned}
A_{N}^{(W)} & =\vec{S}_{\perp} \cdot\left(\hat{p}_{A} \times \mathbf{q}_{\perp}\right) \frac{2\left\langle k_{s}^{2}\right\rangle^{2}}{\left[\left\langle k_{\perp}^{2}\right\rangle+\left\langle k_{s}^{2}\right\rangle\right]^{2}} e^{-\left[\frac{\left\langle k_{\perp}^{2}\right\rangle-\left\langle k_{s}^{2}\right\rangle}{\left\langle k_{\perp}^{2}\right\rangle+\left\langle k_{s}^{2}\right\rangle}\right] \frac{\mathbf{q}_{\perp}^{2}}{2\left\langle k_{\perp}^{2}\right\rangle}} \\
& \times \frac{\sqrt{2 e}}{M_{1}} \frac{\sum_{a b}\left|V_{a b}\right|^{2}\left[-\mathcal{N}_{a}\left(x_{a}\right)\right] f_{a}\left(x_{a}\right) f_{b}\left(x_{b}\right)}{\sum_{a b}\left|V_{a b}\right|^{2} f_{a}\left(x_{a}\right) f_{b}\left(x_{b}\right)}
\end{aligned}
$$

where $\left\langle k_{s}^{2}\right\rangle=M_{1}^{2}\left\langle k_{\perp}^{2}\right\rangle /\left[M_{1}^{2}+\left\langle k_{\perp}^{2}\right\rangle\right]$ and the "_" sign in front of $\mathcal{N}_{a}\left(x_{a}\right)$ is from Eq. (7). If we choose the $\vec{S}_{\perp}$ along the $y$-axis as in Ref. [18], $\vec{S}_{\perp} \cdot\left(\hat{p}_{A} \times \mathbf{q}_{\perp}\right)=q_{T} \cos \left(\phi_{W}\right)$ with $q_{T} \equiv\left|\mathbf{q}_{\perp}\right|$ and azimuthal angle $\phi_{W}$. For our numerical predictions below, we choose $\phi_{W}=0$ and the GRV98LO parton distribution [22] for $f_{q}(x)$ to be consistent with the usage of the TMD distributions of Ref. [18].

In Figs. 1 and 2 , we plot the $A_{N}$ from Eq. (17) at $\sqrt{s}=500 \mathrm{GeV}$. The $W$ asymmetry is peaked at $q_{T} \ll M_{W}$ and is much larger than that of DY production [19]. This is because the $u$ and $d$ Sivers functions have an opposite sign, and they partially cancel each other in their contribution to the DY asymmetry, while they contribute to the $W^{+}$and $W^{-}$separately. The large $W^{-}$
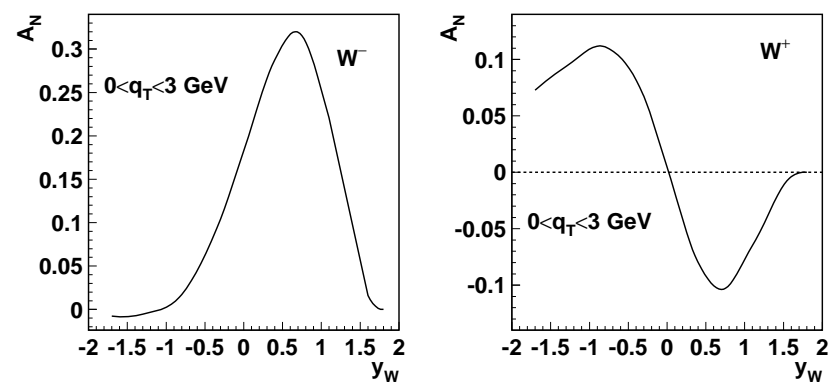

FIG. 1: $A_{N}$ as a function of $W$-boson rapidity.
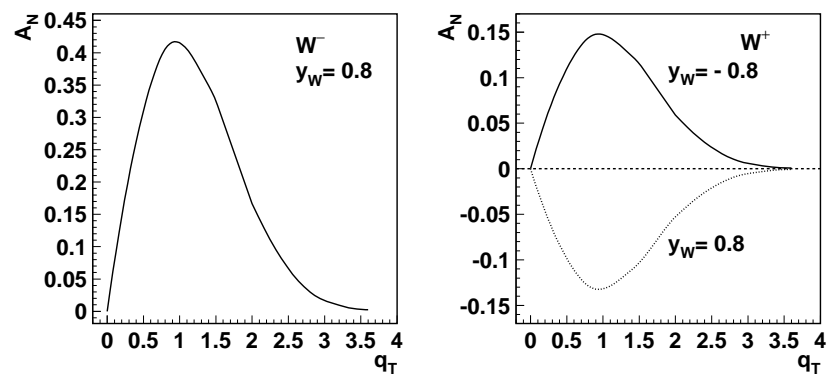

FIG. 2: $A_{N}$ as a function of $W$-boson transverse momentum.

asymmetry is caused by a large $d$ Sivers function [18]. The negative $d$ Sivers function in SIDIS gives the positive $W^{-}$asymmetry. The rapidity dependence in Fig. 1 provides excellent informations for the flavor separation as well as the functional form of the Sivers function if we could reconstruct the $W$ bosons.

After integrating over the momentum of (anti)neutrino from the $W$ decay, we obtain the leading order factorized cross section for the production of leptons of rapidity $y$ and transverse momentum $\mathbf{p}_{\perp}$,

$$
\begin{aligned}
\frac{d \sigma_{A^{\uparrow} B \rightarrow \ell(p)}\left(\vec{S}_{\perp}\right)}{d y d^{2} \mathbf{p}_{\perp}} & =\sum_{a, b}\left|V_{a b}\right|^{2} \int d x_{a} d^{2} \mathbf{k}_{a \perp} \int d x_{b} d^{2} \mathbf{k}_{b \perp} \\
& \times f_{a / A^{\uparrow}}^{\mathrm{DY}}\left(x_{a}, \mathbf{k}_{a \perp}, \vec{S}_{\perp}\right) f_{b / B}\left(x_{b}, k_{b \perp}\right) \\
& \times \frac{1}{16 \pi^{2} \hat{s}}\left|\overline{\mathcal{M}}_{a b \rightarrow \ell}\right|^{2} \delta(\hat{s}+\hat{t}+\hat{u}), \quad(18)
\end{aligned}
$$

where $\hat{s}, \hat{t}$, and $\hat{u}$ are the Mandelstam vaeriables and the leading order partonic scattering amplitude square, $\left|\overline{\mathcal{M}}_{a b \rightarrow \ell}\right|^{2}$, is given by

$$
\frac{8\left(\mathrm{G}_{F} M_{W}^{2}\right)^{2}}{3} \frac{\hat{u}^{2}}{\left(\hat{s}-M_{W}^{2}\right)^{2}+M_{W}^{2} \Gamma_{W}^{2}}
$$

for partonic channels $a b=d \bar{u}, s \bar{u}, \bar{d} u, \bar{s} u$; or by the same one with the $\hat{u}^{2}$ replaced by $\hat{t}^{2}$ for the rest light flavor channels $a b=\bar{u} d, \bar{u} s, u \bar{d}, u \bar{s}$. $\Gamma_{W}$ in Eq. (19) is the $W$ leptonic decay width. Substituting Eq. (6) into Eq. (18), we derive both the spin-averaged and spin-dependent cross sections, from which we evaluate the SSAs of inclusive lepton production from $W$ decay numerically.

In Figs. 3 and 4, we present our predictions for the inclusive lepton asymmetry from the decay of $W$ bosons at 

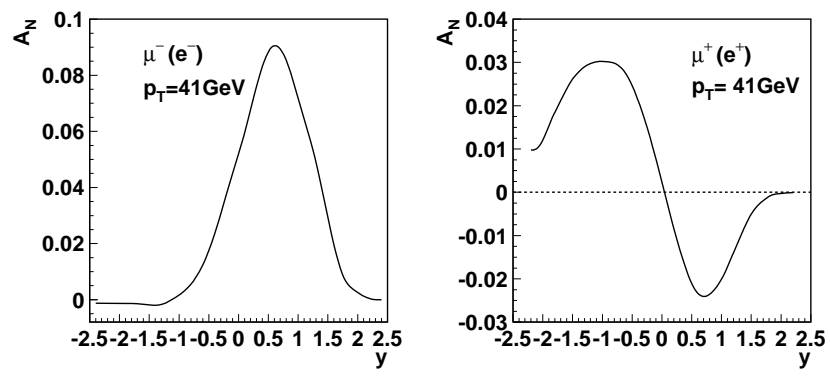

FIG. 3: $A_{N}$ as a function of lepton rapidity.
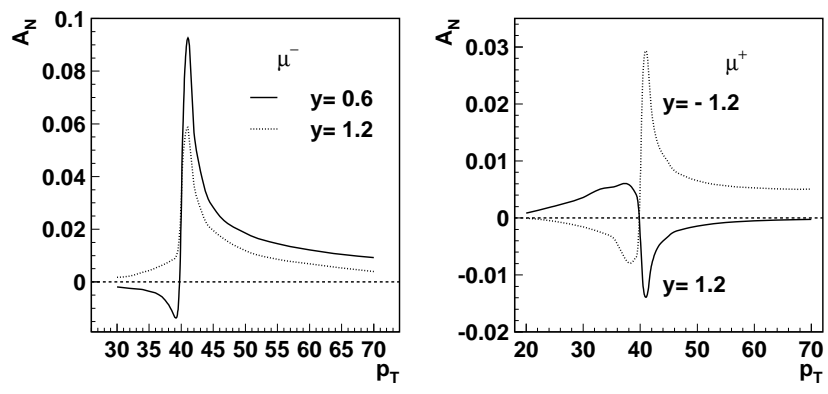

FIG. 4: $A_{N}$ as a function of lepton transverse momentum.

RHIC energy. Although the decay diluted the size of the asymmetry, the lepton inherited all key features of the $W$ asymmetry in Figs. 1 and 2, As shown in Fig. 4, the lepton asymmetry is sharply peaked at $p_{T} \sim 41 \mathrm{GeV}$, which should help control the potential background. The difference in rapidity dependence of the $W^{+}$and $W^{-}$in Fig. 3 provides the excellent flavor separation of the Sivers func- tion, as well as rich information on the functional form. For a good range of rapidity, the lepton asymmetry is measurable at RHIC.

IV. Summary and Conclusions. In summary, we have derived the time-reversal modified universality for both quark and gluon Sivers functions from the parity and time-reversal invariance of the gauge invariant matrix elements that define the TMD parton distributions. We confirm the Collins' prediction for the sign change of the quark Sivers function in SIDIS and in DY 10]. The sign change of the Sivers function in SIDIS and in DY is a natural property of the gauge invariant TMD parton distributions in QCD. Corresponding sign change of the SSAs, if they could be factorized in terms of these TMD parton distributions, is a fundamental prediction of QCD.

We have calculated, in terms of the TMD parton distributions, the SSAs of $W$ production as well as inclusive lepton production from the decay of $W$ bosons in polarized proton-proton collision at RHIC energy. We find that although the asymmetry is diluted from the $W$ decay, the lepton asymmetry is at the level of several percent and measurable for a good range of lepton rapidity at RHIC. Because the lepton asymmetry is sharply peaked at the $p_{T} \sim 41 \mathrm{GeV}$, the potential background could be strongly suppressed. We conclude that this measurable lepton asymmetry at high $p_{T}$ at RHIC is an excellent observable for measuring the Sivers functions of different flavors and for testing the time-reversal modified universality of the Sivers function.

We thank J. Lajoie and F. Wei for helpful discussions. This work was supported in part by the U. S. Department of Energy under Grant No. DE-FG02-87ER40371.
[1] for reviews, see: J. C. Collins, D. E. Soper and G. Sterman, Adv. Ser. Direct. High Energy Phys. 5, 1 (1988).

[2] for reviews, see: J. Pumplin, D. R. Stump, J. Huston, H. L. Lai, P. Nadolsky and W. K. Tung, JHEP 0207, 012 (2002); A. D. Martin, R. G. Roberts, W. J. Stirling and R. S. Thorne, Eur. Phys. J. C 23, 73 (2002).

[3] G. Bunce et al., Phys. Rev. Lett. 36, 1113 (1976).

[4] for reviews, see: U. D'Alesio and F. Murgia, Prog. Part. Nucl. Phys. 61, 394 (2008).

[5] G. L. Kane, J. Pumplin and W. Repko, Phys. Rev. Lett. 41, 1689 (1978).

[6] J. W. Qiu and G. Sterman, AIP Conf. Proc. 223, 249 (1991); Nucl. Phys. B 353, 137 (1991).

[7] A. V. Efremov and O. V. Teryaev, Sov. J. Nucl. Phys. 36, 140 (1982). Phys. Lett. B 150, 383 (1985).

[8] J. W. Qiu and G. Sterman, Phys. Rev. Lett. 67, 2264 (1991), Nucl. Phys. B 378, 52 (1992).

[9] for example, see: Z. B. Kang and J. W. Qiu, Phys. Rev. D 79, 016003 (2009), and references therein.

[10] J. C. Collins, Phys. Lett. B 536, 43 (2002).

[11] D. Boer, P. J. Mulders and F. Pijlman, Nucl. Phys. B 667, 201 (2003).
[12] X. d. Ji, J. P. Ma and F. Yuan, Phys. Lett. B 597, 299 (2004), Phys. Rev. D 71, 034005 (2005).

[13] J. C. Collins and A. Metz, Phys. Rev. Lett. 93, 252001 (2004).

[14] J. Collins and J. W. Qiu, Phys. Rev. D 75, 114014 (2007).

[15] D. W. Sivers, Phys. Rev. D 41, 83 (1990), 43, 261 (1991).

[16] J. C. Collins, Nucl. Phys. B 396, 161 (1993).

[17] X. Ji, J. W. Qiu, W. Vogelsang and F. Yuan, Phys. Rev. Lett. 97, 082002 (2006), Phys. Rev. D 73, 094017 (2006), Phys. Lett. B 638, 178 (2006); Y. Koike, W. Vogelsang and F. Yuan, Phys. Lett. B 659, 878 (2008).

[18] M. Anselmino et al., Eur. Phys. J. A 39, 89 (2009)

[19] J. C. Collins et al., Phys. Rev. D 73, 094023 (2006).

[20] X. d. Ji and F. Yuan, Phys. Lett. B 543, 66 (2002); A. V. Belitsky, X. Ji and F. Yuan, Nucl. Phys. B 656, 165 (2003).

[21] S. J. Brodsky, D. S. Hwang and I. Schmidt, Phys. Lett. B 553, 223 (2003); I. Schmidt and J. Soffer, Phys. Lett. B 563, 179 (2003).

[22] M. Gluck, E. Reya and A. Vogt, Eur. Phys. J. C 5, 461 (1998). 